\title{
Polycrystalline bismuth films: Correlation between grain structure and electron transport
}

\author{
A. S. Fedotov ${ }^{*, 1}$, S. K. Poznyak², L. S. Tsybulskaya ${ }^{2}$, V. G. Shepelevich', I. A. Svito', A. Saad', A. V. Mazanik', \\ and A. K. Fedotov \\ ${ }^{1}$ Belarusian State University, Nezavisimosti Ave. 4, Minsk 220030, Belarus \\ ${ }^{2}$ Research Institute for Physical Chemical Problems, Belarusian State University, Leningradskaya St. 14, Minsk 220030, Belarus \\ ${ }^{3}$ Al-Balqa Applied University, P.O. Box 2041, Salt, Amman 11953, Jordan
}

Received 27 January 2015, revised 7 April 2015, accepted 16 April 2015

Published online 7 May 2015

Keywords bismuth, charge-carrier characteristics, electrophysical properties, grain structure, polycrystalline films

* Corresponding author: email fedotov.alehandro@gmail.com, Phone: +375 2957728 00, Fax: +375 172095445

Grain structure and the temperature dependences of resistivity, magnetoresistance, Hall and Seebeck coefficients measured in the range from 4 to $300 \mathrm{~K}$ were investigated for polycrystalline bismuth films obtained by the melt spinning (MS) and electrochemical deposition (ECD) methods. Charge-carrier concentration and mobilities were calculated assuming the carrier scattering on acoustic deformation potential as the dominant scattering mechanism, parabolicity of holes dispersion law, implying the Lax model for L-band electrons and neglecting the influence of L-band holes on conductivity. The experimental results and calculations have demonstrated that the electrical properties of the Bi films studied are strongly affected by the grain-boundary density.
1 Introduction Thermoelectric materials (TEM) are widely used nowadays for fabrication of thermoelectric generators and Peltier coolers. Bi-based compounds and alloys (together with $\mathrm{Te}, \mathrm{Sb}$, etc.) $[1,2]$ play an important role among industrially used TEM intended for the midtemperature range (up to $\sim 700 \mathrm{~K}$ ). In recent years, there were also a lot of applications for microminiature film type generators/coolers [3] that can operate not only at elevated temperatures, but also at temperatures significantly below room temperature. Such devices can be used for temperature control and stabilisation in the electronic circuits, microchips and other systems like spacecrafts and aircrafts. The films of bismuth-based alloys and compounds can be effectively used for such purposes. Moreover, owing to Bi's huge magnetoresistance, such films could also be applied for creating various kinds of sensors (displacement and magnetic field, etc.).

From a scientific point of view, $\mathrm{Bi}$ is of interest due to its unusual band structure (nonparabolic dispersion law for electrons and overlapping of conduction and valence bands, see Fig. 1), and high mobility of electrons and holes in a wide temperature range $[4,5]$.
Polycrystalline films are the most suitable form of Bi-containing materials for practical applications that is caused by their easier and cheaper fabrication. Currently, however, the properties of the polycrystalline films of $\mathrm{Bi}$ and its alloys are not so well understood in comparison with single-crystalline ones.

The present work is focused on experimental examination of the carrier-transport properties (resistivity, magnetoresistance (MR), Hall and Seebeck effect) of Bi polycrystalline films grown by different methods, as well as estimation of charge-carrier mobility and concentration applying simplified theoretical models.

\section{Experimental}

\subsection{Fabrication and structural characterization}

Three types of samples were used in our research: (i) films prepared by the melt spinning (MS); (ii) electrochemically deposited films (ECD) and (iii) electrochemically deposited films with subsequent annealing (AECD).

Bi of $99.9999 \%$ purity was used for preparing the MS films. Bi was melted and then spilled on the cold surface of a rotating cylinder made of polished copper. The estimated 


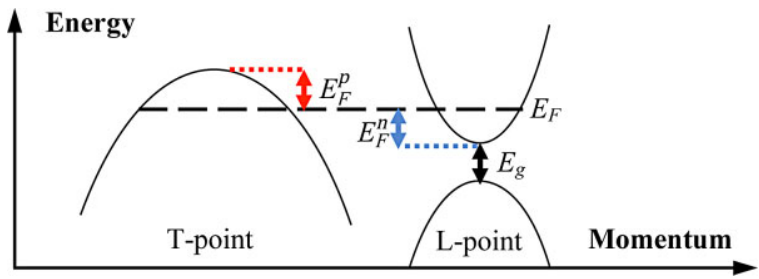

Figure 1 Bismuth band structure.

foil cooling rate was $10^{6}-10^{7} \mathrm{~K} \mathrm{~s}^{-1}[6]$ and their thickness was about $30 \mu \mathrm{m}$.

Electrochemical $\mathrm{Bi}$ deposition was performed from aqueous electrolyte containing $0.174 \mathrm{~mol} \mathrm{l}^{-1} \mathrm{Bi}\left(\mathrm{ClO}_{4}\right)_{3}$ and $3 \mathrm{moll}^{-1} \mathrm{HClO}_{4}$ onto rectangular plates made of a one-sided flexible foil-coated laminate. For electrolyte preparation, bismuth hydroxide powder (Sigma-Aldrich) was completely dissolved in a concentrated perchloric acid solution (65\%) under intensive stirring. Then the solution was diluted to obtain the required concentrations of $\mathrm{Bi}\left(\mathrm{ClO}_{4}\right)_{3}$ and $\mathrm{HClO}_{4}$.

Bismuth films with a thickness of about $70 \mu \mathrm{m}$ were electrodeposited (at room temperature and electrolyte stirring) under a galvanostatic regime with a current density of $2.5 \mathrm{~A} \mathrm{dm}^{-2}$ using a programmable power supply. Bi rod (99.9\%) was used as an anode in the bath. The deposition rate was $65-66 \mu \mathrm{m} \mathrm{h}^{-1}$ and the current efficiency was about $100 \%$. The electrodeposited $\mathrm{Bi}$ coatings were rinsed with distilled water and then were picked off from the substrate by means of substrate bending. Annealing of as-deposited Bi films was carried out at $540 \pm 0.5 \mathrm{~K}$ for $5.5 \mathrm{~h}$ under an inert $(\mathrm{He})$ atmosphere.

The surface morphology and grain structure of the $\mathrm{Bi}$ films were characterized with a LEO $1455 \mathrm{VP}$ scanning electron microscope and a PANalytical Empyrean diffractometer. Grain size identification was performed with Oxford's Tango software.

2.2 Electrical measurements The temperature and field dependences of resistivity, magnetoresistance, Hall effect and thermoelectromotive force (thermo-EMF) were measured in the 4-300 K temperature range and in magnetic fields up to $8 \mathrm{~T}$. Measurements were performed using a closed-cycle measuring system produced by Cryogenic Ltd. (London).

For measurements of resistivity $\rho$, Hall coefficient $R_{\mathrm{H}}$ and MR, the rectangular samples $(2 \times 12 \times 15 \mathrm{~mm})$ with two current, two potential and two Hall contacts deposited by ultrasound soldering of indium were fabricated. Movable bronze clamping probes were used for thermo-EMF measurements. The sample was placed in a special measuring cell involving thermometers, magnetic-field sensors and heaters to control the temperature. The measuring probes were inserted into a superconducting solenoid inside of the cryostat and connected to the measuring circuit. Sample temperature during measurements of electrical properties was controlled with special Cernox sensors and a LakeShore 331 measuring controller providing the temperature measurement and stabilisation with an accuracy of $0.005 \mathrm{~K}$.
All measurements were done under a DC regime. Resistivity and Hall effect were measured by the 4-probe potentiometric method and 4 switching method, respectively. Current through the sample was passed using a Keithley 6430 SourceMeter, which allows to set current with $0.05 \%$ precision and to measure sample resistance in the range from $100 \mu \Omega$ to $20 \mathrm{G} \Omega$. Magnetoresistance (MR) was measured in magnetic fields, perpendicular to the current direction. The temperature difference on the sample during thermo-EMF measurements was equal to $1.5 \mathrm{~K}$.

The relative error for DC measurements did not exceed $5 \%$ for resistivity (this was determined mainly by the error of sample sizes and interprobe distance measurements), $0.1-0.2 \%$ for MR and $0.5-1 \%$ for $R_{\mathrm{H}}$. The measurement error of Seebeck coefficient $S$ depends on temperature and a temperature gradient. As a result, the relative error of measurement does not exceed $2 \%$ above $200 \mathrm{~K}$ and $10 \%$ at low temperatures $(4-10 \mathrm{~K})$.

Electrical measurements were performed using three samples from each type of film demonstrating a good reproducibility (error within 5\%) of the obtained data.

\section{Results and Discussion}

3.1 Structure Top-view SEM images (Fig. 2) demonstrate that the linear size of Bi grains $l_{\mathrm{G}}$ for the MS films varies from 5 to $15 \mu \mathrm{m}$, while for the as-prepared ECD films the grains are much smaller $\left(l_{\mathrm{G}} \sim 0.5-1.5 \mu \mathrm{m}\right)$.

The linear density of grain boundaries (number of boundaries per unit length) determined from SEM images was estimated as $0.11 \mu \mathrm{m}^{-1}$ for the MS films, $1.4 \mu \mathrm{m}^{-1}$ for the ECD films and $0.04 \mu \mathrm{m}^{-1}$ for the AECD films.

$\mathrm{X}$-ray diffraction studies show that the MS and ECD films reveal pronounced $\langle 012\rangle$ texture, whereas annealing of the ECD samples leads to its disappearance (Fig. 3).

3.2 Electrical properties As can be seen from Fig. 4a, the ECD and MS samples display completely

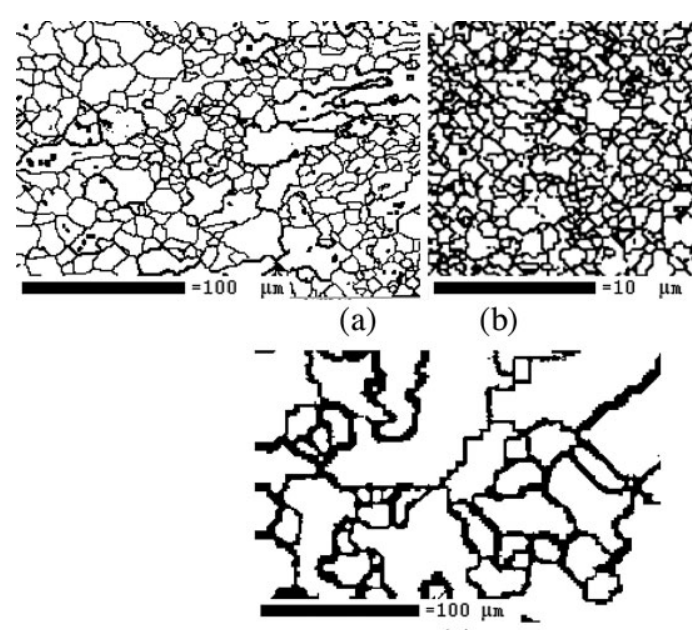

(c)

Figure 2 Grain boundaries according to SEM in (a) the MS, (b) ECD and (c) AECD films. 


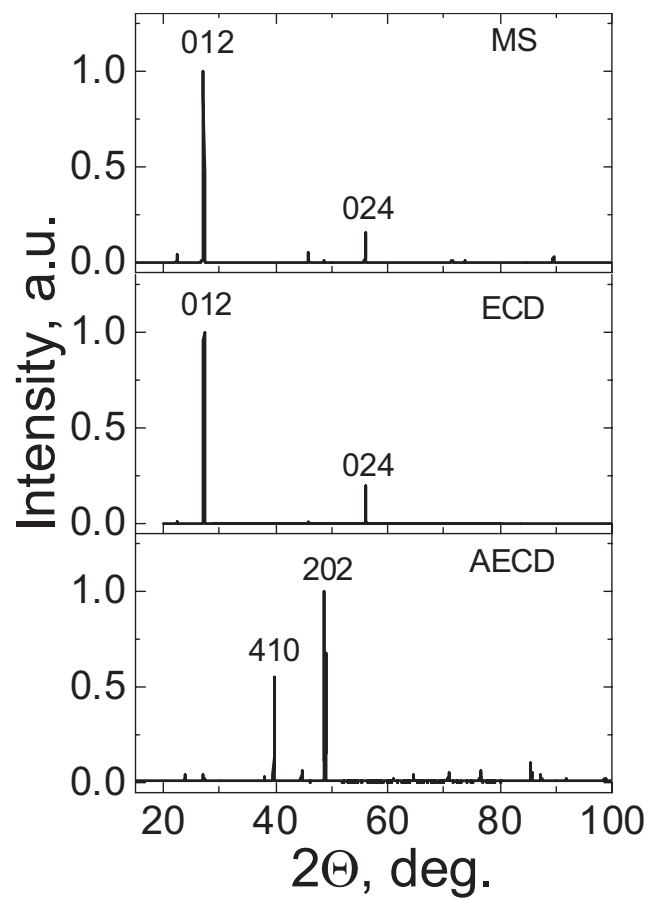

Figure 3 X-ray diffraction patterns for the MS, ECD and AECD films.

different behavior of $\rho(T)$ dependences in the studied temperature range. Typical $\rho(T)$ curves for the ECD films look similar to those for classical semiconductors (when $(\mathrm{d} \rho / \mathrm{d} T)<0)$, whereas for the MS samples it is close to metallic behaviour with a positive sign of $\mathrm{d} \rho / \mathrm{d} T$. After annealing, the ECD samples demonstrate a $\rho(T)$ dependence practically coincident with that for the MS films.

The Seebeck coefficient for all types of the films studied is negative and the modulus of $S(T)$ shows a maximum at $T \sim 200 \mathrm{~K}$ (Fig. 4b). At room temperature, the absolute values of $S$ for the MS films are slightly smaller compared with those for ECD and AECD films. This difference could be tentatively explained by the fact that the $\langle 012\rangle$ texture is less favourable to achieve high thermo-EMF values in comparison to the case of randomly oriented grains [7], as well as by a higher density of grain boundaries in the ECD films compared to MS ones having the same texture [8].

The Hall coefficient is also negative and decreases with temperature (Fig. 4c) that is in agreement with the results obtained by other researchers [7, 9].

To investigate the carrier-transport properties of the $\mathrm{Bi}$ films in more detail, we also measured magnetoresistance in a wide range of temperatures and magnetic fields. The experiments have shown that at $4 \mathrm{~K}$ relative $\operatorname{MR}\left(\Delta \rho / \rho_{\mathrm{O}}=[\rho(B)-\rho(0)] / \rho(0)\right)$ values in a magnetic field of $B=8 \mathrm{~T}$ reach about 4500 for the AECD films, while for the MS films $\Delta \rho / \rho_{0}$ is about 1600 and for the as-deposited ECD films it is about 12. In addition, $\operatorname{MR}(B, T)$ dependences in the low magnetic field range were also studied to estimate the temperature dependencies of carrier concentration and mobility. As follows
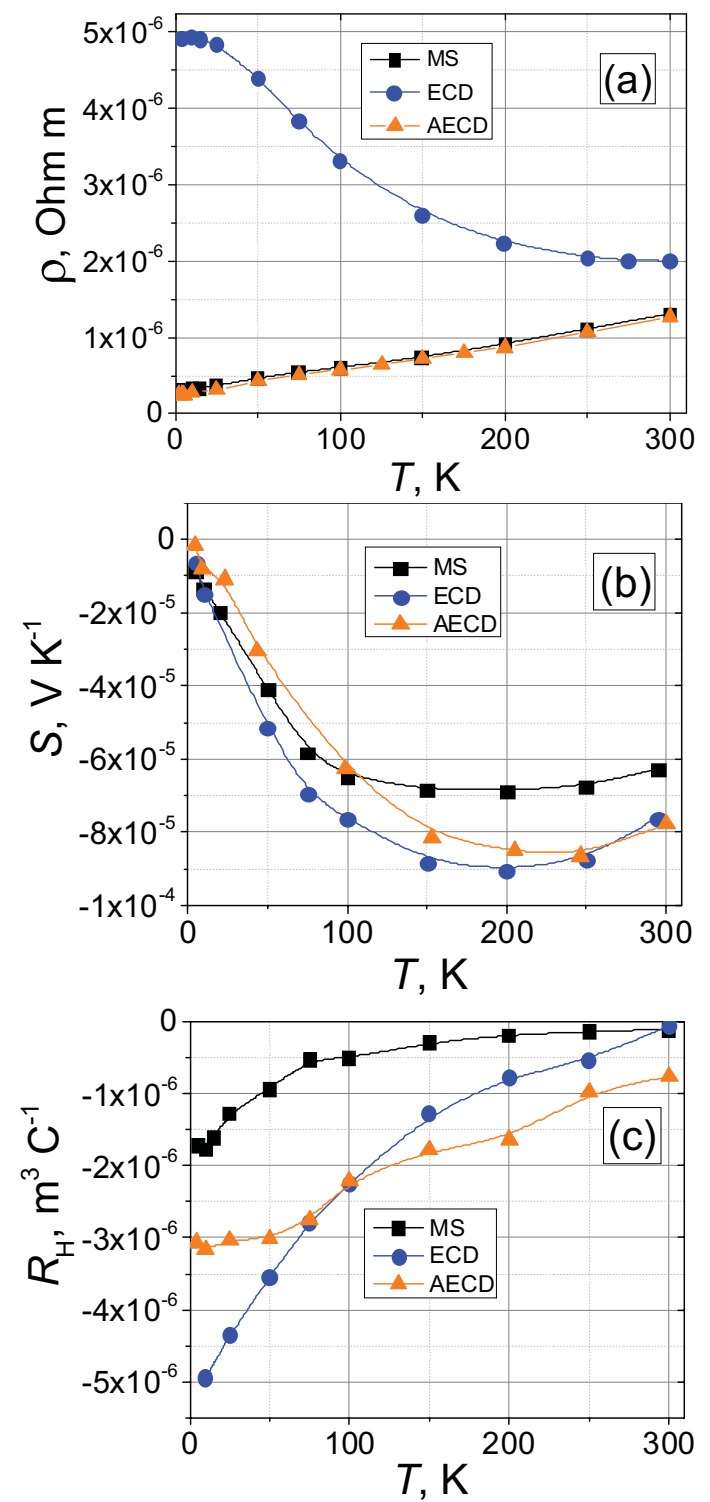

Figure 4 Temperature dependences of (a) resistivity $\rho(T)$, (b) Seebeck coefficient $S(T)$ in zero magnetic field and (c) Hall constant $R_{\mathrm{H}}(T)$ at $B=0.25 \mathrm{~T}$ for the Bi films prepared by different methods.

from Fig. 5, for all temperatures the low- $B \operatorname{MR}(B)$ curves are linear on a double-logarithmic scale; for the AECD and MS large-grained films they lie very close to each other, while the ECD films show a significantly lower MR effect. The difference between the MR values for the large- (AECD and MS) and fine-grained (ECD) films increases with the temperature fall. In the context of the above-mentioned grain-size difference, such behaviour evidences a strong influence of grain-boundary density on the MR effect.

Parallelism of the low- $B$ curves in Fig. 5 with a tangent close to 2 for all temperatures means parabolicity of $\operatorname{MR}(B)$ dependences. Such behaviour (together with the MR increase with decreasing temperature) can be easily 

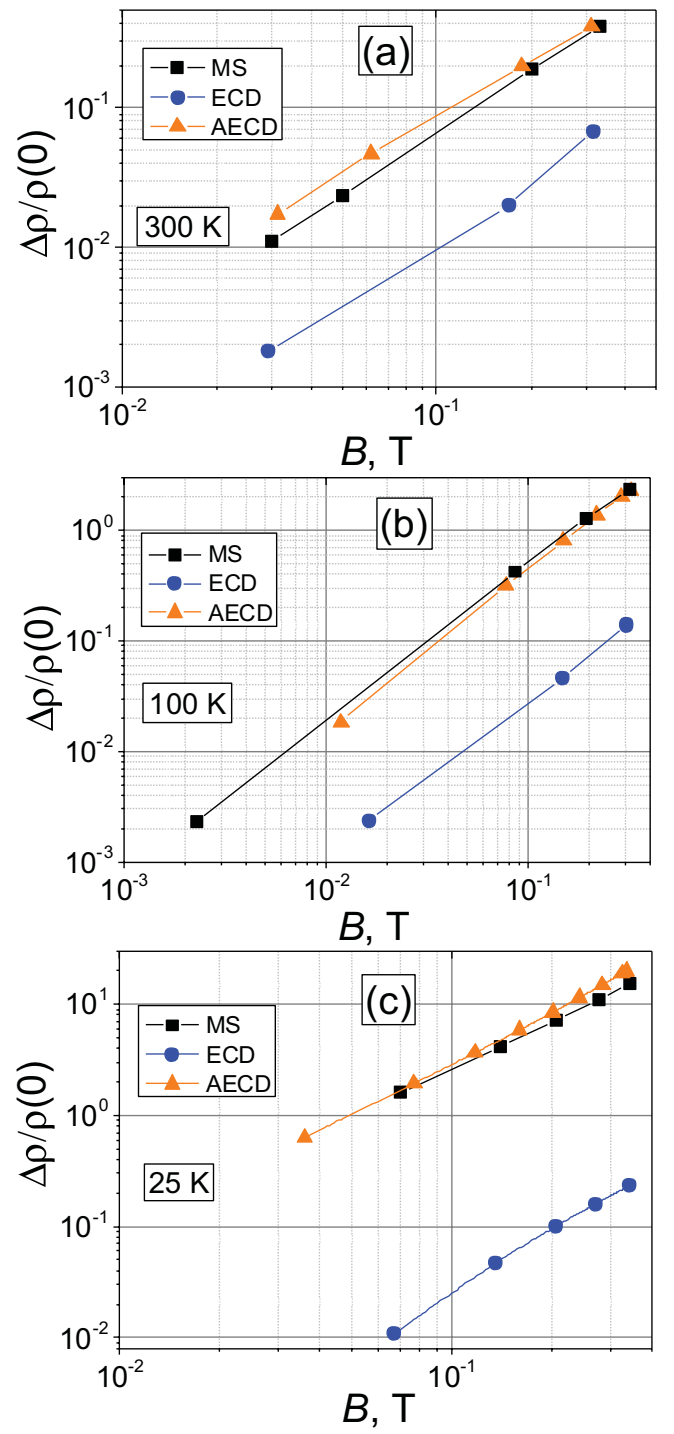

Figure 5 Field dependences of relative magnetoresistance $[\rho(B)-\rho(0)] / \rho(0)$ at (a) $T=300 \mathrm{~K}$, (b) $T=100 \mathrm{~K}$ and (c) $T=25 \mathrm{~K}$.

explained by the Lorentz mechanism of the magnetoresistance effect. Actually, the Lorentz force leads to significant curving of charge-carrier trajectories only if electrons and holes are weakly scattered on phonons and defects, i.e. when mean free path is high enough [10]. At high temperatures, phonons act as main mean free path limiters determining a decrease in relative MR with increasing temperature. At low temperatures, the role of scattering on grain boundaries increases, leading to a more pronounced dependence of the MR values on the grainboundary density in the films.

Note that the measured MR values of the MS and AECD films are not in contradiction with results reported in [11, 12], while the MR of ECD film is significantly smaller due to the smaller grain size.

Below, the main equations and assumptions needed for estimation of carriers concentrations and mobilities are considered. In accordance with [13], in a low-field range the $\operatorname{MR}(B)$ curves can be fitted by the known expression

$$
\begin{aligned}
\frac{\Delta \rho}{\rho_{\mathrm{B}} B^{2}}= & \frac{9 \pi}{16}\left(\frac{\left(3 r+\frac{3}{2}\right) !}{\left(\left(r+\frac{3}{2}\right) !\right)^{3}} \frac{p \mu_{\mathrm{p}}^{3}+n \mu_{\mathrm{n}}^{3}}{p \mu_{\mathrm{p}}+n \mu_{\mathrm{n}}}\right. \\
& \left.-\left(\frac{\left(2 r+\frac{3}{2}\right) !}{\left(\left(r+\frac{3}{2}\right) !\right)^{2}} \frac{p \mu_{\mathrm{p}}^{2}+n \mu_{\mathrm{n}}^{2}}{p \mu_{\mathrm{p}}+n \mu_{\mathrm{n}}}\right)^{2}\right),
\end{aligned}
$$

where $B$ is the magnetic field induction, $r$ is the scattering factor, $n$ and $p$ are the electron and hole concentrations and $\mu_{\mathrm{n}}$ and $\mu_{\mathrm{p}}$ are the electron and hole mobilities.

The conductivity in zero magnetic field can be expressed as follows:

$$
\sigma=\sigma_{\mathrm{n}}+\sigma_{\mathrm{p}}=e n \mu_{\mathrm{n}}+e p \mu_{\mathrm{p}}
$$

In the vanishing field limit, the effective Hall coefficient can be expressed through the Hall factor $r_{\mathrm{H}}$ and the chargecarrier mobilities and concentrations [14]:

$$
R_{\mathrm{H}}=\frac{r_{\mathrm{H}}}{e} \frac{p \mu_{\mathrm{p}}^{2}-n \mu_{\mathrm{n}}^{2}}{\left(p \mu_{\mathrm{p}}+n \mu_{\mathrm{n}}\right)^{2}} .
$$

The Hall factor is usually close to 1 and slightly depends on the scattering factor $r$ in Eq. (1) [13].

As pure $\mathrm{Bi}$ can be considered as an intrinsic semiconductor, the equality of concentrations

$$
n=p
$$

can be applied for calculations.

The effective Seebeck coefficient can be expressed with Matthiessen's rule [5, 9, 15]

$$
S=\frac{S_{\mathrm{p}} \sigma_{\mathrm{p}}+S_{\mathrm{n}} \sigma_{\mathrm{n}}}{\sigma_{\mathrm{p}}+\sigma_{\mathrm{n}}},
$$

where $S_{\mathrm{n}}$ and $S_{\mathrm{p}}$ are partial Seebeck coefficients for electrons and holes, $\sigma_{\mathrm{n}}=n e \mu_{\mathrm{n}}$ and $\sigma_{\mathrm{p}}=p e \mu_{\mathrm{p}}$ are the electron and hole contributions to conductivity, respectively. We calculated the partial Seebeck coefficients in an isotropic approximation using a simple two-band model assuming that only Tpoint holes contribute to conductivity. The partial coefficient for holes with parabolic band dispersion law $S_{\mathrm{p}}$ can be expressed as

$$
S_{\mathrm{p}}=\frac{k_{\mathrm{B}}}{e}\left(\frac{(r+5 / 2) F_{r+5 / 2}\left(\frac{E_{\mathrm{f}}^{\mathrm{p}}}{k_{\mathrm{B}} T}\right)}{(r+3 / 2) F_{r+1 / 2}\left(\frac{E_{\mathrm{f}}^{\mathrm{p}}}{k_{\mathrm{B}} T}\right)}-\frac{E_{\mathrm{f}}^{\mathrm{p}}}{k_{\mathrm{B}} T}\right),
$$


where $E_{\mathrm{f}}^{\mathrm{p}}$ is the Fermi energy for holes (Fig. 1), $k_{\mathrm{B}}$ is the Boltzmann constant and $F_{j}\left(E_{\mathrm{f}} /\left(k_{\mathrm{B}} T\right)\right)$ is the Fermi integral expressed as

$$
F_{j}\left(\frac{E_{\mathrm{f}}}{k_{\mathrm{B}} T}\right)=\frac{1}{j !} \int_{0}^{\infty} \frac{x^{j}}{e^{x-\frac{E_{\mathrm{f}}}{k_{\mathrm{B}} T}}+1} \mathrm{~d} x .
$$

To take into account a nonparabolicity of the electron energy dispersion law, the Lax model was used for describing the partial Seebeck coefficient for electrons $[5,15]$

$$
S_{\mathrm{n}}=-\frac{k_{\mathrm{B}}}{e}\left(\frac{I_{r+5 / 2}\left(\frac{E_{\mathrm{f}}^{\mathrm{n}}}{k_{\mathrm{B}} T}\right)}{I_{r+3 / 2}\left(\frac{E_{\mathrm{f}}^{\mathrm{n}}}{k_{\mathrm{B}} T}\right)}-\frac{E_{\mathrm{f}}^{\mathrm{n}}}{k_{\mathrm{B}} T}\right),
$$

where $E_{\mathrm{f}}^{\mathrm{n}}$ is the Fermi energy for electrons (Fig. 1) and

$$
\begin{aligned}
I_{s}\left(\frac{E_{\mathrm{f}}}{k_{\mathrm{B}} T}\right)= & \int_{0}^{\infty} x^{s}\left(1+\frac{x k_{\mathrm{B}} T}{E_{\mathrm{g}}}\right)^{s+3 / 2} e^{x-\frac{E_{\mathrm{f}}}{k_{\mathrm{B}} T}} \\
& \times\left(1+e^{x-\frac{E_{\mathrm{f}}}{k_{\mathrm{B}} T}}\right)^{-2} \mathrm{~d} x .
\end{aligned}
$$

In our calculations we used the temperature dependence of L-point bandgap $E_{g}$ and Fermi energies for holes and electrons reported in [5].

We have obtained the temperature dependences of $n(p), \mu_{\mathrm{n}}$ and $\mu_{\mathrm{p}}$ using two different equation systems: either Eqs. (1)-(4) (system A with the equation for Hall coefficient) or Eqs. (1), (2), (4) and (5) (system B with the equation for Seebeck coefficient). The scattering factor was chosen equal to $-1 / 2$ (acoustic phonon scattering) in the equation for Seebeck coefficient. Experimental measurements of resistivity, magnetoresistance, Seebeck and Hall coefficients are being used as input data for those equation systems. The $n(T), \mu_{\mathrm{n}}(T)$ and $\mu_{\mathrm{p}}(\mathrm{T})$ dependencies calculated on the basis of the A and B systems for different samples are presented in Figs. 6 and 7.

As can be seen from Fig. 6, the concentrations of electrons (holes) increase monotonically with temperature and are very close to the values known from the literature for non-doped Bi single crystals [7]. In so doing, the values of concentrations calculated from the equation systems A and $\mathrm{B}$ were matched with high precision (less than $0.1 \%$ ). The charge-carrier concentrations calculated for $\mathrm{Bi}$ films of different types differ slightly. This fact proves the correctness of the analysis because the concentration of charge carriers should not vary for thick films of the same chemical composition and crystalline structure.

The mobility of carriers for the fine-grained ECD films is slightly dependent on temperature at $T<100 \mathrm{~K}$, while for the MS and AECD samples this dependence is much stronger for the whole temperature range studied (Fig. 7).

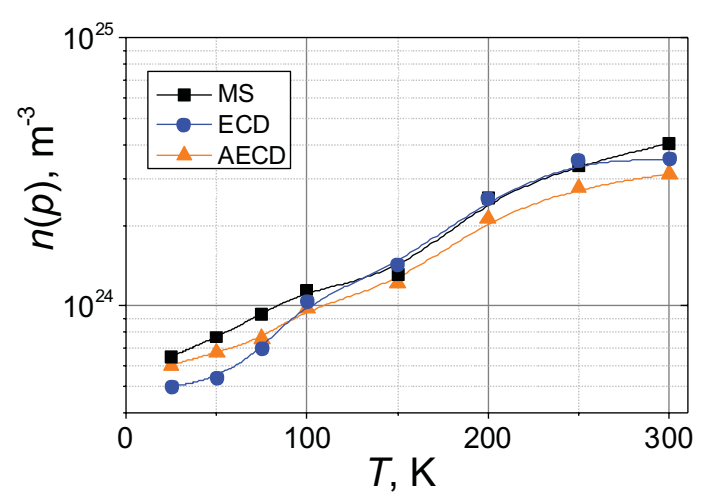

Figure 6 Temperature dependence of the charge-carrier concentration for the studied samples of polycrystalline Bi films.
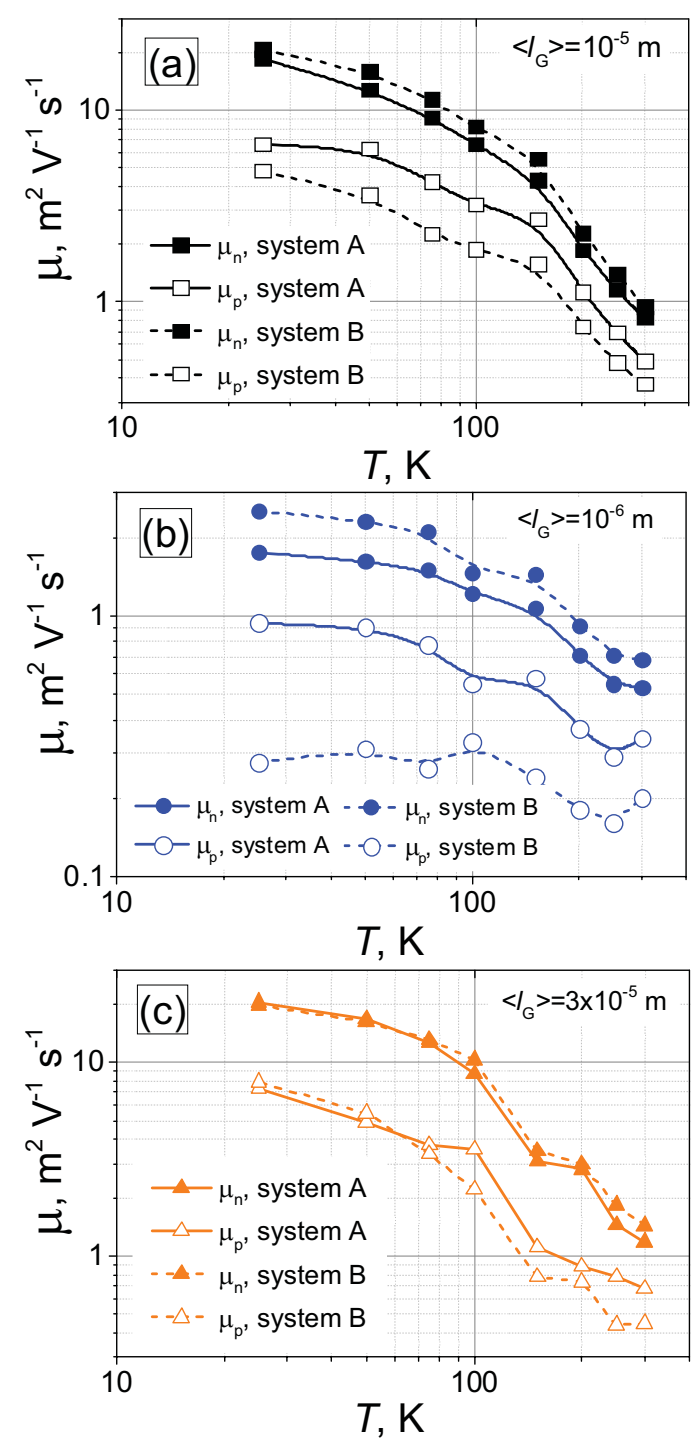

Figure 7 Temperature dependences of electron and hole mobilities calculated using the equation systems A [Eqs. (1)-(4)] and B [Eqs. (1), (2), (4) and (5)] for (a) the MS, (b) ECD and (c) AECD samples. $\left\langle l_{\mathrm{G}}\right\rangle$ is an order of the average grain size. 
This difference can be understood taking into account the fact that the linear density of grain boundaries is much higher in the ECD films (Section 3.1) and, hence, at low temperatures grain-boundary scattering may be considered as a dominant scattering mechanism for these samples. The slight temperature dependence of carriers scattering on structural defects as compared with phonon scattering $[15,16]$ explains the insignificant mobility variation with temperature in the ECD films.

Also, note that for the ECD films the divergence between mobilitiy values calculated using the equation systems A (Hall coefficient) and B (Seebeck coefficient; dominance of acoustic phonon scattering is supposed) is significantly larger in comparison to that for the MS and the AECD samples (Fig. 7). This fact is additional corroboration of the phonon-scattering dominance in the MS and AECD films, as well as the enhanced influence of grain-boundary scattering in the ECD ones.

The obtained $n(T), \mu_{\mathrm{n}}(T)$ and $\mu_{\mathrm{p}}(T)$ dependencies allow us to easily explain the difference in the temperature behaviour of resistivity for the large- and fine-grained films (Fig. 4a). Resistivity is known to be inversely proportional to the concentration and mobility of charge carriers. The concentration increases approximately eightfold in the range from 25 to $300 \mathrm{~K}$ for all samples (Fig. 6). For the fine-grained ECD samples, the mobility decreases with temperature only by 2-3 times, whereas for the large-grained MS and AECD films - more than by one order of magnitude. As for the ECD samples, mobility decreases slowly with increasing temperature (because the efficiency of scattering on grain boundaries is weakly sensitive to temperature), the temperature dependence of resistivity will be determined mainly by the temperature dependence of carrier concentrations. At the same time, for the large-grained MS and AECD films the carrier mobilities decrease rapidly with increasing temperature, because scattering on grain boundaries plays a much smaller role as compared with strong temperature-dependent phonon scattering. Therefore, the temperature dependence of resistivity for the large-grained samples is determined mainly by the temperature dependences of mobilities.

4 Conclusions The experimental study of resistivity, magnetoresistance, Hall and Seebeck coefficients in the temperature range from 4 to $300 \mathrm{~K}$, as well as calculations based on these data have shown a correlation between electric properties and grain structure of the polycrystalline bismuth films obtained by the melt spinning and electrochemical deposition methods. Charge-carrier concentrations and mobilities were calculated on the assumption of scattering on the acoustic deformation potential as the dominant mechanism of carriers scattering and using the Lax model with a nonparabolic dispersion law for electrons. These estimations allowed determination of the role of boundary scattering of carriers and explained the temperature dependences of resistivity in terms of the scattering on grain boundaries.

\section{References}

[1] D. M. Rowe (ed.), Thermoelectrics Handbook: Macro to Nano (Taylor and Francis, Boca Raton 2006), pp. 30-130-14.

[2] O. Caballero-Calero, P. Díaz-Chao, B. Abad, C. V. Manzano, M. D. Ynsa, J. J. Romero, M. Rojo, and M. S. MartínGonzález, Electrochim. Acta 123, 117 (2014).

[3] M. Tan, Y. Deng, and Y. Hao, Energy 70, 143 (2014).

[4] G. Jezequel, J. Thomas, and I. Pollini, Phys. Rev. B 56, 6620 (1997).

[5] D. Nakamura, M. Murata, H. Yamamoto, Y. Hasegawa, and T. Komine, J. Appl. Phys. 110, 053702 (2011).

[6] W. J. Boettinger, J. H. Perepezko, and H. H. Liebermann, Rapidly Solidified Alloys: Processes, Structures, Properties, Applications (Marcel Dekker, Inc, New York, 1993), pp. $68-73$.

[7] C. F. Gallo, B. S. Chandrasekhar, and P. S. Sutter, J. Appl. Phys. 11, 268 (1963).

[8] K. Kishimoto and T. Koyanagi, J. Appl. Phys. 92, 2544 (2002).

[9] S. Kochowski and A. Opilski, Thin Solid Films 48, 345 (1978)

[10] M. H. Jeun, K. I. Lee, and W. Y. Lee, J. Korean Phys. Soc. 46, S80 (2005).

[11] F. Y. Yang, K. Liu, K. Hong, D. H. Reich, P. C. Searson, and C. L. Chien, Science 284, 1335 (1999).

[12] J. Chang, H. Kim, J. Han, M. H. Jeon, and W. Y. Lee, J. Appl. Phys. 98, 023906 (2005).

[13] K. Seeger, Semiconductor Physics: An Introduction (Springer, Berlin, 1991), p. 65.

[14] J. N. Zemel, Nondestructive Evaluation of Semiconductor Materials and Devices (Springer US, New York, 1979), p. 84.

[15] B. Lax, J. G. Mavroides, H. J. Zeiger, and R. J. Keyes, Phys. Rev. Lett. 5, 241 (1960).

[16] J. Heremans and O. P. Hansen, J. Phys. C, Solid State Phys. 12, 3483 (1979). 